\title{
Momentum dependence of the superconducting gap in $\mathrm{NdFeAsO}_{0.9} \mathrm{~F}_{0.1}$ single crystals measured by angle resolved photoemission spectroscopy
}

\author{
Takeshi Kondo, ${ }^{1}$ A. F. Santander-Syro,${ }^{2,3}$ O. Copie,${ }^{4}$ Chang Liu, ${ }^{1}$ M. E. Tillman, ${ }^{1}$ \\ E. D. Mun, ${ }^{1}$ J. Schmalian, ${ }^{1}$ S. L. Bud'ko, ${ }^{1}$ M. A. Tanatar, ${ }^{1}$ P. C. Canfield, ${ }^{1}$ and A. Kaminski ${ }^{1}$ \\ ${ }^{1}$ Ames Laboratory and Department of Physics and Astronomy, Iowa State University, Ames, Iowa 50011, USA \\ ${ }^{2}$ Laboratoire Photons Et Matière, UPR-5 CNRS, \\ ESPCI, 10 rue Vauquelin, 75231 Paris cedex 5, France \\ ${ }^{3}$ Labratoire de Physique des Solides, UMR-8502 CNRS, \\ Université Paris-Sud, Bât. 510, 91405 Orsay, France \\ ${ }^{4}$ Unité Mixte de Physique CNRS/Thales, Route départementale 128, 91767 Palaiseau Cedex, France
}

(Dated: November 5, 2018)

\begin{abstract}
We use angle resolved photoemission spectroscopy (ARPES) to study the momentum dependence of the superconducting gap in $\mathrm{NdFeAsO}_{0.9} \mathrm{~F}_{0.1}$ single crystals. We find that the $\Gamma$ hole pocket is fully gapped below the superconducting transition temperature. The value of the superconducting gap is $15 \pm 1.5 \mathrm{meV}$ and its anisotropy around the hole pocket is smaller than $20 \%$ of this value - consistent with an isotropic or anisotropic s-wave symmetry of the order parameter. This is a significant departure from the situation in the cuprates, pointing to the possibility that the superconductivity in the iron arsenic based system arises from a different mechanism.
\end{abstract}

PACS numbers: 79.60.-i, 74.25.Jb, 74.70.-b

The gap function is the single most important quantity that can be used to reveal the pairing mechanism of a superconductor. It's symmetry and shape in momentum space are intimately linked to the many body interactions that are responsible for the creation of the Cooper pairs. The recent discovery of superconductivity in iron arsenic based materials [1, 2, 3, 4, has initiated intense experimental [5, 6, 7, 8, 9, 10, 11, 12, 13, 14, 15, and theoretical [16, 17, 18, 19, 20, 21] effort. The undoped, non-superconducting systems of both oxygen containing $\mathrm{RFeAsO}(\mathrm{R}=\mathrm{La}, \mathrm{Nd}, \mathrm{Sm})$ and oxygen free $\mathrm{AFe}_{2} \mathrm{As}_{2}$ $(\mathrm{A}=\mathrm{Ba}, \mathrm{Sr}, \mathrm{Ca})$, display structural [1, 22, 23, 24, 25. and magnetic [6, 26] phase transitions at elevated temperatures. Doping with $\mathrm{RFeAsO}$ with fluorine (electron doping) or $\mathrm{AFe}_{2} \mathrm{As}_{2}$ with potassium (hole doping) leads to a suppression of the transition temperature and the emergence of superconductivity [1, 2, 3, 4, 23]. Perhaps most remarkably, it has recently been discovered [27] that undoped $\mathrm{CaFe}_{2} \mathrm{As}_{2}$ can also be made superconducting by applying a very modest amount of external pressure $\sim 5$ kbar. One of the most pressing questions is whether the mechanism of the superconductivity in this system is similar to that in the classical low temperature superconductors or the cuprate high temperature superconductors, or if this is a completely new route to the superconducting state. The large atomic masses of iron and arsenic, combined with the very high critical temperature, seem to exclude conventional phonon mediated pairing. It should be pointed out again, though the superconducting phase transition appears to be in close proximity to a suppressed structural phase transition. This immediately brings the possibility of phonons and their role in Cooper pair formation back to the forefront. The fact that in pure $\mathrm{CaFe} 2 \mathrm{As} 2$ this can be seen as function of modest pressures, raises the possible role of phonons even further, even though the lack of light elements in the common FeAs-layers requires a subtly enhanced electron-phonon coupling between Fe-3d electrons and vibrations of out of plane atoms. A knowledge of the symmetry and shape in momentum space of the superconducting order parameter is essential for constructing the correct model of the pairing mechanism. A number of different scenarios have been proposed to explain the mechanism of the superconductivity in this system 16, 17, 18, 19, 20, 21, with predictions about the symmetry of the order parameter ranging from isotropic and anisotropic $s$-wave to $d$-wave and $p$-wave. Angle resolved photoemission spectroscopy (ARPES) is an excellent tool to address this question. Even though this technique is not sensitive to the phase of the order parameter, it can directly measure its absolute value as a function of momentum via the superconducting gap. In most cases, one can deduce the character of the order parameter from the symmetry of the superconducting gap.

Here we report on ARPES measurements of the superconducting gap in $\mathrm{NdFeAsO}_{0.9} \mathrm{~F}_{0.1}$ single crystals. First and most importantly we find that the Fermi surface is fully gapped - that is, there are no nodes in the order parameter at the Fermi momenta. The magnitude of the superconducting gap is $15 \pm 1.5 \mathrm{meV}$ and is comparable to that from our previous measurements within the experimental uncertainty (error bars and doping) [11. Our data also limits the possible anisotropy of the superconducting gap to, at most 20\%. Although the results have sizable error bars, if the gap is indeed anisotropic, the data are consistent with minima located along $\Gamma-\mathrm{M}$, that is at a $45^{\circ}$ angle to the Fe-Fe bond. Our results are also consistent with an isotropic $s$-wave symmetry of 
the order parameter and exclude ordinary $p$-wave and $d$ wave symmetries. However, an anisotropic s-wave state, where gap nodes are located between distinct Fermi surface sheets is also consistent with our data, in particular as such a state creates a small anisotropy of the superconducting gap

High pressure synthesis of samples with the nominal composition of $\mathrm{NdFeAsO}_{0.9} \mathrm{~F}_{0.1}$ was carried out in a cubic, multianvil press, with an edge length of $19 \mathrm{~mm}$ from Rockland Research Corporation. Stoichiometric amounts of $\mathrm{NdFe}_{3} \mathrm{As}_{3}, \mathrm{Nd}_{2} \mathrm{O}_{3}, \mathrm{NdF}_{3}$ and $\mathrm{Nd}$ were pressed into a pellet with a mass of approximately $0.5 \mathrm{~g}$ and placed inside of a BN crucible with an inner diameter of 5.5 $\mathrm{mm}$. The synthesis was carried out at a pressure of 3.3 GPa. The temperature was increased, over one hour, from room temperature to $1350-1400^{\circ} \mathrm{C}$ and then held there for 8 hours before being quenched to room temperature. The pressure was then released and the sample was removed mechanically. This synthesis produced a high density pellet that contained large grains (up to 300 $\times 200 \mu \mathrm{m}$ in cross section [28]) of superconducting $\left(T_{\mathrm{c}}\right.$ $\sim 53 \mathrm{~K}) \mathrm{NdFeAsO}_{0.9} \mathrm{~F}_{0.1}$ as well as non-superconducting $\mathrm{NdOFeAs.} \mathrm{In} \mathrm{addition} \mathrm{there} \mathrm{are} \mathrm{inclusions} \mathrm{of} \mathrm{FeAs} \mathrm{and}$ $\mathrm{Nd}_{2} \mathrm{O}_{3}$. Magneto optical measurements [29] indicate that on average the samples are over $50 \%$ superconducting. The single crystals were extracted mechanically from the pellet. Samples with a size of $\sim 200 \times 200 \times 50 \mu$ m were cleaved in situ yielding a flat mirror-like surface. ARPES experiments were carried out using a Scienta SES2002 hemispherical analyzer attached to the PGM beam line at the Synchrotron Radiation Center (SRC), Wisconsin. The profile of the photon beam on the sample surface was slightly elliptical with a mean diameter smaller than $\sim 100 \mu \mathrm{m}$. All spectra were measured at $20 \mathrm{~K}$ using $22 \mathrm{eV}$ photon. As a reference for the Fermi energy, we used the spectral edge position of evaporated $\mathrm{Au}$ in electrical contact with the sample. The momentum resolution was set at $0.13^{\circ}$ and the energy resolution was set at $\sim 16 \mathrm{meV}$ confirmed by measuring energy width between $90 \%$ and $10 \%$ intensity positions of Au Fermi edge.

We have determined the orientation of the sample and the location of the Fermi surface from both the ARPES intensity and momentum distribution curves (MDC). A plot of the ARPES intensity integrated over $\pm 20 \mathrm{meV}$ about the Fermi energy as a function of momentum 30, 31 is shown in Fig. 1(e). It reveals a Fermi surface consistent with our previous report [11] for samples from a different batch. Now we focus on two different momentum cuts (A and B) along the high symmetry directions, $\Gamma-\mathrm{X}$ and $\Gamma-\mathrm{M}$, respectively. Their location in the Brillouin zone is indicated by arrows in Fig.1(e). The ARPES intensity along these two cuts is shown in Figure $1 \mathrm{a}$ and $\mathrm{b}$ as a function of momentum and energy for $T=20 \mathrm{~K}$, deep in the superconducting state. At first glance these plots exhibit all the characteristics of a sample in the superconducting state: a buildup of
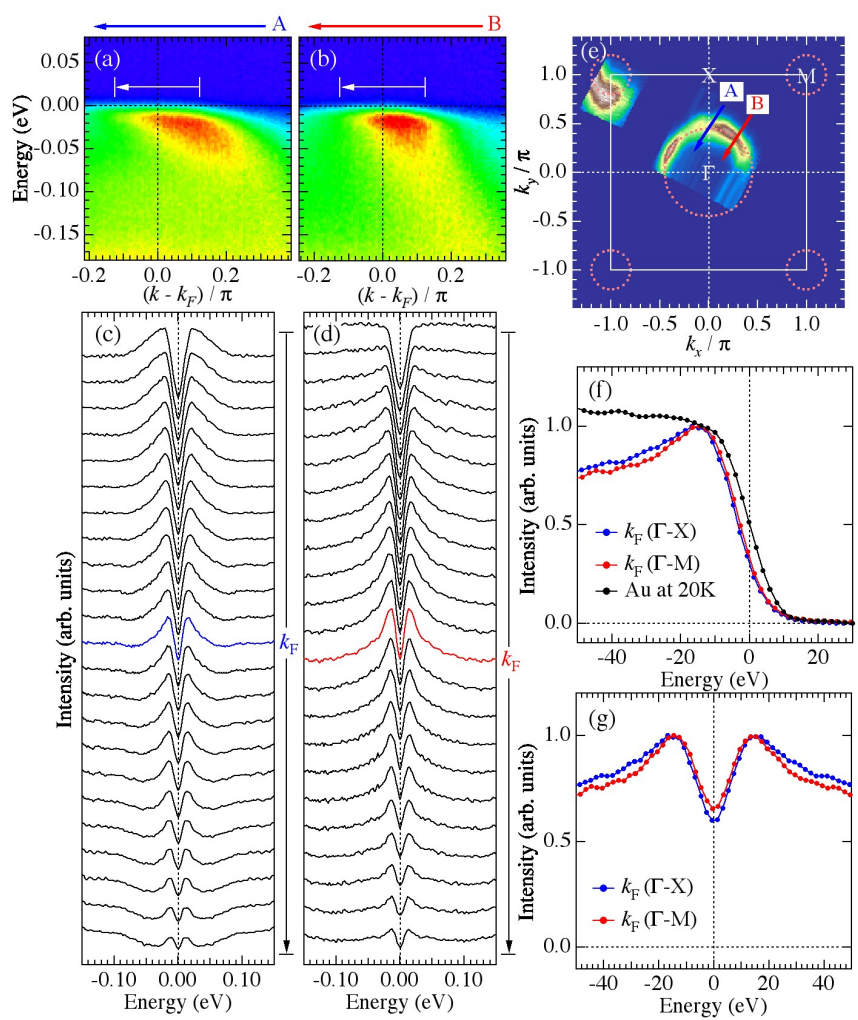

FIG. 1: (Color online) The superconducting gap along the high symmetry directions measured at $T=20 \mathrm{~K}$. (a)-(b) ARPES intensity map along the $\Gamma-\mathrm{X}$ and $\Gamma-\mathrm{M}$ directions, respectively (directions shown in panel (e)). (c)-(d) Energy distribution curves (EDCs) for panels (a) and (b), respectively. The momentum range is indicated by the white arrows in panels (a) and (b). The colored curves mark the EDC at the Fermi momenta. (e) ARPES intensity map as a function of $k_{x}$ and $k_{y}$ momentum, integrated within $20 \mathrm{meV}$ of the Fermi energy. Bright areas mark the location of the Fermi surface. (f) Comparison of the gap magnitude along $\Gamma-\mathrm{X}$ and $\Gamma-\mathrm{M} .(\mathrm{g})$ Symmetrized EDCs from the data in panel (f).

intensity just below the chemical potential with a characteristic arc shape that arises from particle-hole mixing 32 - a hallmark of a pairing induced energy gap. To confirm this observation of a superconducting gap we used the symmetrization method 33] on the raw EDC data: EDCs are reflected about the Fermi energy and added to the unreflected ones. This removes the effects of the Fermi function and enables us to immediately identify the presence of an energy gap by the appearance of two peaks separated by a dip, as opposed to a single peak at the chemical potential when there is no superconducting gap. Figure 1 (c) and (d) show the symmetrized EDCs corresponding to the data in panel (a) and (b), respectively. We determined the Fermi wavevector $\left(k_{\mathrm{F}}\right)$ from the peak position of the MDCs at the Fermi energy. The opening of a superconducting gap is clearly observed in both directions: two sharp peaks do not merge into a single peak but remain separated at and beyond $k_{\mathrm{F}}$ due 

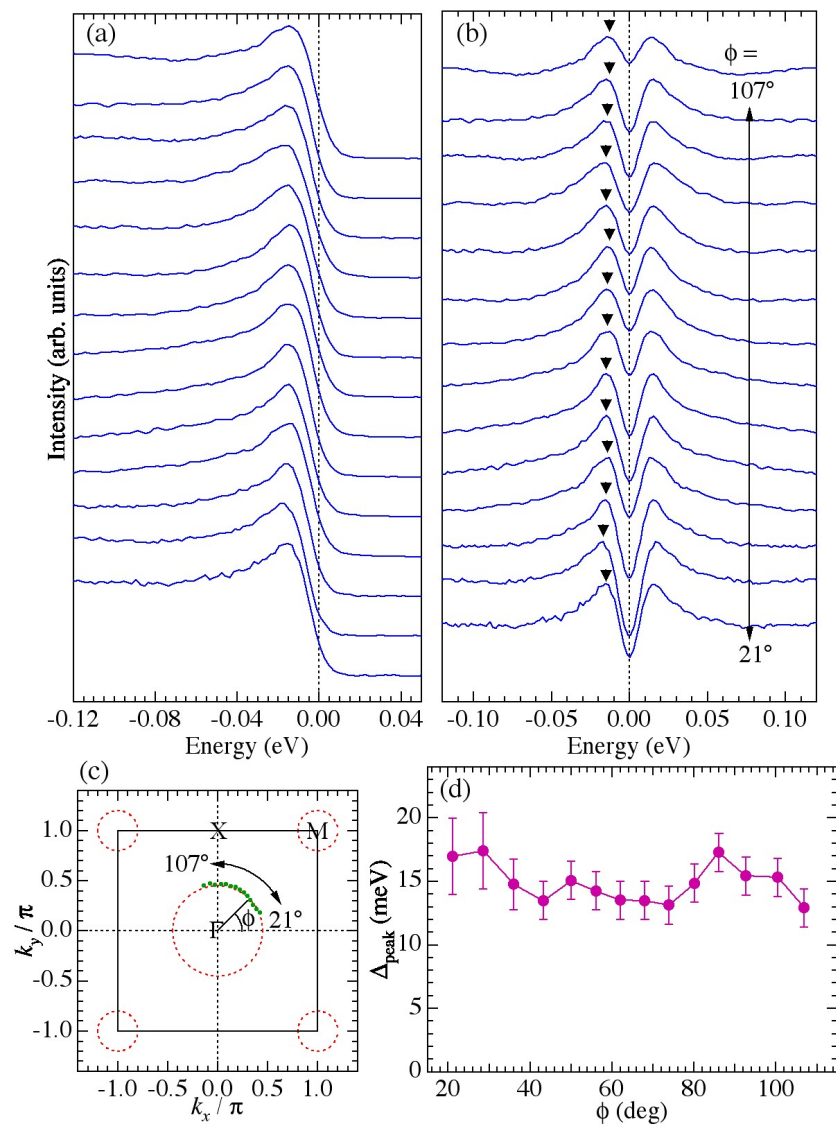

FIG. 2: (Color online) The magnitude of the superconducting gap along the $\Gamma$ hole-pocket. (a) raw- and (b) symmetrizedEDCs at the Fermi crossing momenta marked by green dots in panel (c), where the definition of the $\phi$ angle is shown. (d) The value of the superconducting gap extracted from the data in panel (b) using the coherent peak position method.

to particle hole mixing [32]. The width of the coherent peak $(\sim 20 \mathrm{meV})$ is mostly limited by the experimental energy resolution. Note that the behavior of the coherent peak contrasts with the rapid broadening of the spectral line shape towards the higher binding energy due to an increase in the electron scattering rate [34]. In order to compare the gap sizes along the two different directions, we plot the EDCs in Fig.1 (f) and the corresponding symmetrized EDCs in Fig.1 (g). The superconducting gap can be easily estimated from the EDC data by evaluating the energy position of the coherent peak vs. the Fermi energy. We found that the superconducting gap has a similar value $\sim 17 \pm 1.5 \mathrm{meV}$ in both directions. The gap along $\Gamma-\mathrm{X}$ appears slightly bigger than along the $\Gamma-\mathrm{M}$ direction (all data points of the peak are further from the chemical potential and the dip is larger), however we cannot conclude this with great certainty given the experimental error bars.

We measured the ARPES spectra at a several Fermi momenta points around the $\Gamma$-centered hole pocket $(\Gamma$ -

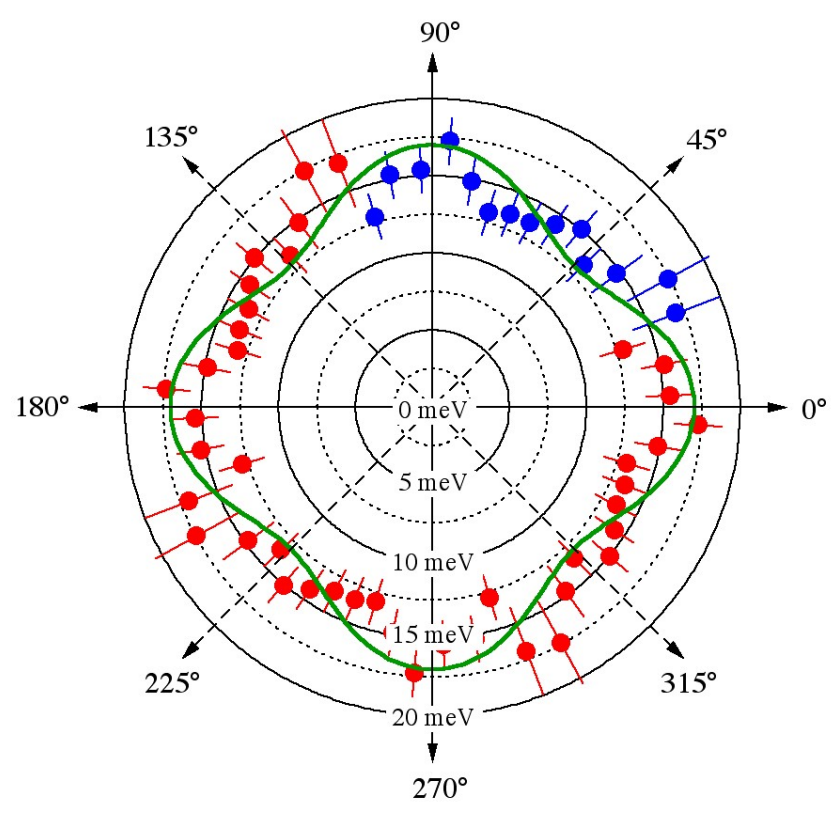

FIG. 3: (Color online) Magnitude of the superconducting gap around the $\Gamma$-pocket in polar coordinates. It is clear from this graph that the gap is always open, with a magnitude $\left(\Delta_{\text {peak }}\right)$ the varies between $\sim 13 \mathrm{meV}$ and $\sim 18 \mathrm{meV}$, indicating conventional $s$-wave or slightly anisotropic $s$-wave behavior at the $\Gamma$-pocket. The green line indicates a model with a slight gap anisotropy of $20 \%$ that would still be consistent with this data.

pocket), in order to obtain the symmetry of the superconducting gap (Fig. 2). We covered a wide range of the Fermi surface angle $\left(21^{\circ} \leq \phi \leq 107^{\circ}\right)$, which is roughly a quarter of the Fermi surface as shown in Fig. 2(c). The EDCs and the corresponding symmetrized ones measured at each Fermi momentum point are shown in Fig. 2(a) and (b), respectively. We determined the size of the energy gap using the previously mentioned coherent peak position method. The results are shown in Fig. 2(d) with the summary shown in polar coordinates in Fig 3. To give a better sense of the gap symmetry we reflected the results from one quadrant into the other three quadrants using the crystal symmetry axes. We use blue symbols to mark the measured data and red ones to indicate the data points that are a reflection of the actual data. The superconducting gap is never zero around the Fermi surface, indicating a lack of nodes. This excludes simple $p$ - or $d$-wave pairing scenarios, which have nodes on the Fermi surface. In the simplest scenario, our data is consistent with isotropic $s$-wave behavior, however we cannot exclude the possibility of a small anisotropy being present of order of $20 \%$ due to the finite error bars. This would also be consistent with a pairing state with nodes of the gap-function between distinct sheets of the Fermi surface. Such an anisotropic gap is indicated in Fig. 3 by the green line which shows a $20 \%$ anisotropy and lies within 
the error bars of our experiment 28]. Microscopically, a pairing state with nodes between the Fermi surface sheets is most likely based upon a non-phononic mechanism with strong interband scattering. A likely candidate mechanism is based upon paramagnon fluctuations with an in plane wave vector close to $(\pi / a, \pi / a)$ [16], i.e. with a wave vector equal or close to that of the ordered spin density wave state of the undoped systems [35]. On the other hand, a fully isotropic s-wave state would make the electron-phonon mechanism a viable candidate. To distinguish between the subtle signatures of this limited subset of models, an additional ARPES study with significantly smaller error bars is necessary. However, our data already clearly excludes pairing states with gap nodes on the Fermi surface.

In conclusion, we used angle resolved photoemission spectroscopy to study the momentum dependence of the superconducting gap in the newly discovered electrondoped oxypnictide superconductor $\mathrm{NdFeAsO}_{0.9} \mathrm{~F}_{0.1}$. We found a nodeless superconducting gap in the hole pocket around $\Gamma(0,0)$. The gap magnitude $(\sim 15 \mathrm{meV})$ is almost constant around the Fermi surface within a variation of less than $20 \%(\sim 1.5 \mathrm{meV})$ - if the gap is indeed anisotropic. Our results exclude $p$-wave and $d$-wave pairing states with nodes of the gap on the Fermi surface, but are consistent with both an isotropic gap or a state where nodes are located between distinct Fermi surface sheets. The latter, unconventional pairing state implies a small anisotropy of the superconducting gap, consistent with the data presented in this letter. Our results are in general agreement with the conclusions of penetration depth measurements [28, 36].

We acknowledge useful discussions with R. Prozorov and M. A. Tanatar. We thank Helen Fretwell for useful remarks and corrections. Work at Ames Laboratory was supported by the Department of Energy - Basic Energy Sciences under Contract No. DE-AC02-07CH11358. The Synchrotron Radiation Center is supported by NSF DMR 9212658. AFSS thanks LPEM for financial support.

Note added: After completion of this work we become aware of a draft manuscript reporting measurements on the hole doped relative, $\mathrm{Ba}_{1-x} \mathrm{~K}_{x} \mathrm{Fe}_{2} \mathrm{As}_{2}$ [37] which also concluded the presence of node-less gap with a consistent magnitude and limits (error bars) on its possible anisotropy.
[1] Yoichi Kamihara, Takumi Watanabe, Masahiro Hirano, and Hideo Hosono, J. Am. Chem. Soc. 130, 3296 (2008).

[2] Hiroki Takahashi et al., Nature(London) 453, 376 (2008)

[3] Ren Zhi-An et al., Chin. Phys. Lett. 25, 2215 (2008).

[4] M. Rotter, M. Tegel and D. Johrendt, arXiv:condmat/0805.4630 (2008).

[5] T. Y. Chen, Z. Tesanovic, R. H. Liu, X. H. Chen and C. L. Chien, /nature07081 (2008).

[6] Clarina de la Cruz et al., Nature 453, 899(2008)

[7] F. Hunte et al., Nature 453, 903 (2008),

[8] Xiaowen Jia, et al., arXiv:cond-mat/0806.0291 (2008)

[9] Haiyun Liu et al., arXiv:cond-mat/0805.3821 (2008)

[10] L. X. Yang et al., arXiv:cond-mat/0806.2627 (2008)

[11] C. Liu et al., arXiv:cond-mat/0806.2147 (2008)

[12] C. Liu et al., arXiv:cond-mat/0806.3453 (2008)

[13] K. Hashimoto et al., arXiv:cond-mat/0806.3149 (2008)

[14] L. X. Yang et al., arXiv:cond-mat/0806.2627 (2008)

[15] Haiyun Liu et al., arXiv:cond-mat/0806.4806 (2008)

[16] I. I. Mazin, D. J. Singh, M. D. Johannes and M. H. Du, arXiv:cond-mat/0803.2740 (2008).

[17] K. Kuroki et al., arXiv:cond-mat/0803.3325 (2008).

[18] Xi Dai, Zhong Fang, Yi Zhou and F. C. Zhang, arXiv:cond-mat/0803.3982 (2008)

[19] P. A. Lee and X. G. Wen, arXiv:cond-mat/0804.1739 (2008).

[20] Z. J. Yao, J. X. Li and Z. D. Wang, arXiv:condmat/0804.4166 (2008).

[21] Fa Wang, Hui Zhai, Ying Ran, Ashvin Vishwanath and Dung-Hai Lee, arXiv:cond-mat/0807.0498 (2008)

[22] M. Rotter et al., arxiv:cond-mat/0805.4021 (2008)

[23] N. Ni et al., arXiv:cond-mat/0806.1874, (2008)

[24] J. -Q. Yan et al., arXiv:cond-mat/0806.2711, (2008)

[25] N. Ni et al., arXiv:cond-mat/0806.4328, (2008)

[26] X. F. Wang et al., arXiv:cond-mat/0806.2452, (2008)

[27] Milton S. Torikachvili, Sergey L. Bud'ko, Ni Ni and Paul C. Canfield, arXiv:cond-mat/0807.0616 (2008)

[28] C. Martin et al., arXiv:cond-mat/0807.0876

[29] R. Prozorov et al., arXiv:cond-mat/0805.2783 (2008)

[30] H. M. Fretwell et al., Phys. Rev. Lett. 84, 4449 (2000).

[31] J. Mesot et al., Phys. Rev. B 63, 224516 (2001).

[32] J. C. Campuzano et al., Phys. Rev. B 53, R14737 (1996):

[33] M. R. Norman et al., Nature 392, 157 (1998).

[34] K. Haule, J. H. Shim and G. Kotliar, Phys. Rev. Lett. 100, 226402 (2008).

[35] Y. Qiu et al., arXiv:cond-mat/0805.1062 (2008)

[36] L. Malone et al., arXiv:cond-mat/0806.3908 (2008)

[37] L. Zhao et al., arXiv:cond-mat/0807.0398 (2008) 